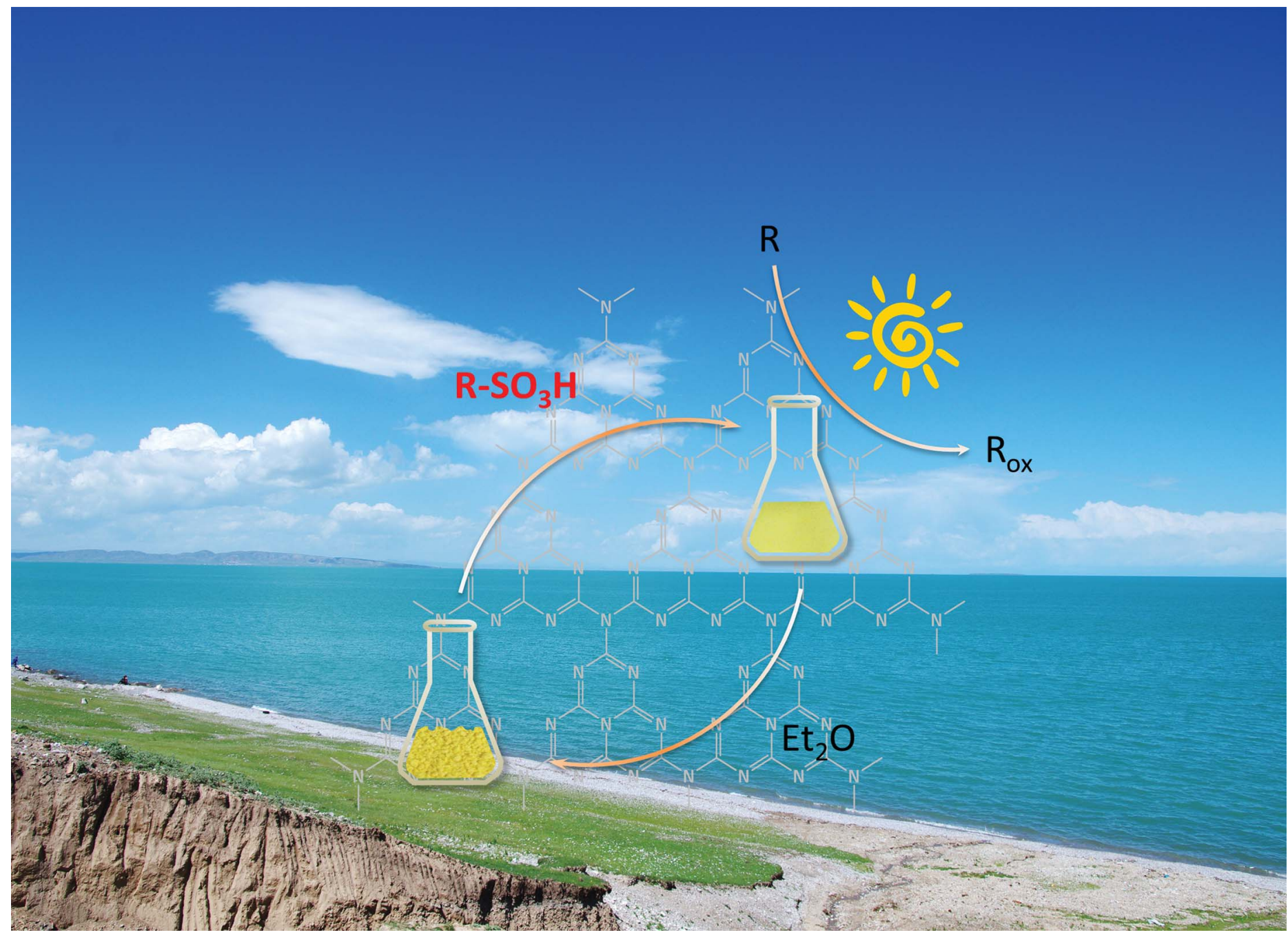

\section{Showcasing research from Professor Yuanjian Zhang's} laboratory (Southeast University, China) and Professor Haibo Ma's laboratory (Nanjing University, China)

Dissolution and homogeneous photocatalysis of polymeric carbon nitride

Polymeric carbon nitride $(\mathrm{CN})$ is a promising (photo)catalyst towards heterogeneous reactions and optoelectronic biosensing. By following prototype of enzymes, making all catalytic sites accessible via homogeneous routes is envisioned to maximize activities, but hindered by the poor solubility of $\mathrm{CN}$. We report the unusual dissolution of $\mathrm{CN}$ in environmental-friendly methanesulfonic acid. The homogeneous $\mathrm{CN}$ showed 10 -time boosted activity, meanwhile heterogeneous catalysts hallmark (facile separation and recycling) was kept. It opens new vista of $\mathrm{CN}$ in homogeneous catalysis and offers a successful example of polymeric catalysts in bridging homo/heterogeneous catalysis.

\section{As featured in:}

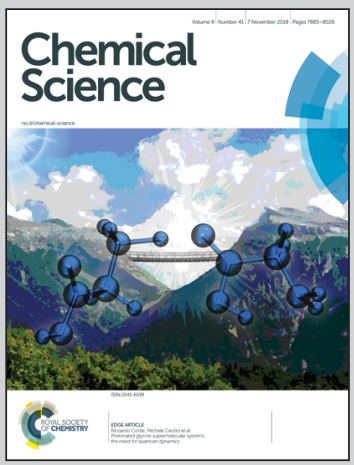

See Haibo Ma, Yuanjian Zhang et al., Chem. Sci., 2018, 9, 7912. 
Check for updates

Cite this: Chem. Sci., 2018, 9, 7912

๑ All publication charges for this article have been paid for by the Royal Society of Chemistry

\title{
Dissolution and homogeneous photocatalysis of polymeric carbon nitride $\uparrow$
}

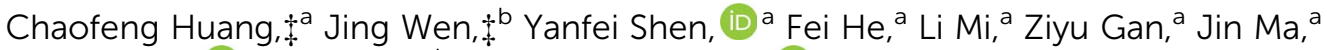 \\ Songqin Liu, (D) ${ }^{a}$ Haibo Ma*b and Yuanjian Zhang (D) *a
}

As a metal-free conjugated polymer, carbon nitride $(\mathrm{CN})$ has attracted tremendous attention as a heterogeneous (photo)catalyst. By following the example of enzymes, making all of the catalytic sites accessible via homogeneous reactions is a promising approach toward maximizing $\mathrm{CN}$ activity, but hindered due to the poor solubility of $\mathrm{CN}$. Herein, we report the dissolution of $\mathrm{CN}$ in environmentally friendly methanesulfonic acid, and homogeneous photocatalysis (two biomimetic/pharmaceutical photocatalytic oxidation reactions) driven by $\mathrm{CN}$ for the first time with the activity boosted up to 10 times compared to the heterogeneous counterparts. Moreover, facile recycling and reusability, the hallmarks of heterogeneous catalysts, were kept for the homogeneous $\mathrm{CN}$ photocatalyst via reversible precipitation using poor solvents. This study opens a new vista for $\mathrm{CN}$ in homogeneous catalysis and offers a successful example of a polymeric catalyst that bridges the gap between homo/heterogeneous catalysis.

Received 29th August 2018

Accepted 2nd October 2018

DOI: $10.1039 / \mathrm{c} 8 \mathrm{sc} 03855 \mathrm{~d}$

rsc.li/chemical-science

disclosed to dissolve bulk poly(triazine imide) (PTI $\cdot \mathrm{LiBr})$-based $\mathrm{CN}$ with controllable luminescent properties. ${ }^{4}$ As an innovative alternative, exfoliating bulk $\mathrm{CN}$ into nanosheets greatly boosted its photocatalytic activity. ${ }^{5}$ Similarly, in the early days of graphene and carbon nanotube research, their insolubility in all solvents remained as one of the biggest impediments to the realization of their potential applications. ${ }^{6}$ Therefore, seeking more general solvents to solubilize $\mathrm{CN}$ and perform homogeneous catalysis would be fascinating for the subsequent research of $\mathrm{CN}$.

stimulated substantial interest in the conversion of organic molecules and solar-to-chemical energy conversion and beyond, e.g. in optoelectronic biosensing, due to its unique electronic structure and surface properties. ${ }^{2}$ By following the example of enzymes, making all of the catalytic sites accessible via homogeneous reactions is a promising approach toward maximizing $\mathrm{CN}$ activity. However, the poor solubility of $\mathrm{CN}$, ascribed to the interlayer van der Waals forces, ${ }^{3}$ makes the use of $\mathrm{CN}$ for homogeneous catalysis difficult. Concentrated $\mathrm{H}_{2} \mathrm{SO}_{4}$ was reported as the first solvent for $\mathrm{CN}$, but the harsh conditions hindered the further application of this approach. Polar aprotic solvents, such as dimethyl sulfoxide (DMSO), have been

ajiangsu Engineering Laboratory of Smart Carbon-Rich Materials and Device, Jiangsu Province Hi-Tech Key Laboratory for Bio-Medical Research, School of Chemistry and Chemical Engineering, Medical School, Southeast University, Nanjing 211189, China. E-mail: Yuanjian.Zhang@seu.edu.cn

${ }^{b}$ School of Chemistry and Chemical Engineering, Nanjing University, Nanjing 210023, China.E-mail: haibo@nju.edu.cn

$\dagger$ Electronic supplementary information (ESI) available. See DOI: $10.1039 / \mathrm{c} 8 \mathrm{sc} 03855 \mathrm{~d}$

\$ C. Huang and J. Wen contributed equally to the work.

\section{Results and discussion}

Herein, we report the dissolution of $\mathrm{CN}$ in $\mathrm{CH}_{3} \mathrm{SO}_{2} \mathrm{OH}$ (MSA), an environmentally friendly solvent. As controls, other sulfonic acid group-containing solvents, including $\mathrm{ClSO}_{2} \mathrm{OH}$ (ClSA), $\mathrm{HOSO}_{2} \mathrm{OH}\left(\mathrm{H}_{2} \mathrm{SO}_{4}\right)$, and $\mathrm{C}_{2} \mathrm{H}_{5} \mathrm{OSO}_{2} \mathrm{CH}_{3}$ (EMS), were also investigated. Taking two photocatalytic oxidations as examples, up to 10-fold faster catalytic kinetics of homogeneous CN were achieved relative to those of the heterogeneous one. Moreover, due to its capability for reversible dissolution and recovery using good and poor solvents, $\mathrm{CN}$ could be facilely separated from the homogeneous system and reused, thus combining the advantages of both homogeneous and heterogeneous catalysts as shown in Fig. 1a.

The starting $\mathrm{CN}$ with interlinked heptazine-based units (Fig. S1-S3†) and a stacked lamellar texture (Fig. S4 $\dagger$ ) was prepared by a conventional thermal polymerization of dicyandiamide at $550{ }^{\circ} \mathrm{C}$ in air. The dissolution ability of CN in MSA and control solvents at room temperature is demonstrated in 
(a)

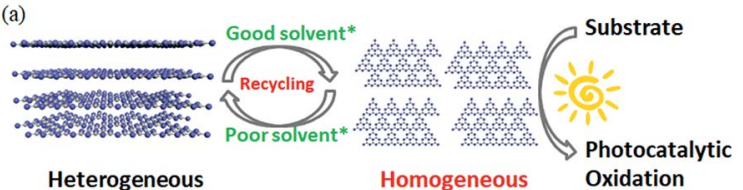

Heterogeneous

*Good solvent: $\mathrm{CH}_{3} \mathrm{SO}_{2} \mathrm{OH}$; Poor solvent $\mathrm{CH}_{3} \mathrm{CH}_{2} \mathrm{OCH}_{2} \mathrm{CH}_{3}$
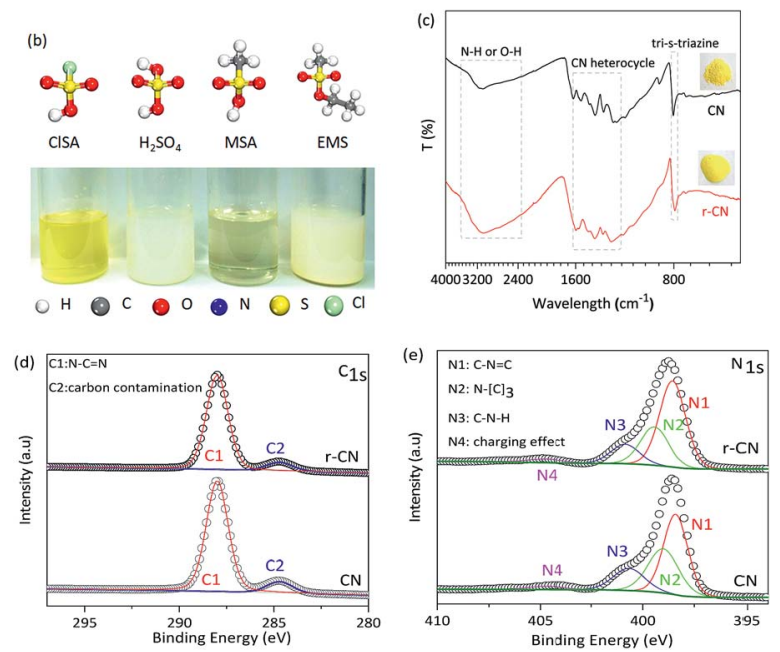

Fig. 1 (a) Bridging homo/heterogeneous photocatalysis. (b) A photo of $\mathrm{CN}$ in sulfonic acid group-containing solvents $\left(50 \mathrm{mg} \mathrm{mL}^{-1}\right)$ at room temperature, and a ball-and-stick model of the solvents. Bulk CN was only completely dissolved in MSA. (c) FT-IR, (d) XPS C 1s and (e) N 1s spectra of bulk $\mathrm{CN}$ and that recovered from the MSA solution ( $\mathrm{r}-\mathrm{CN})$. Inset: photos of $\mathrm{CN}$ and $\mathrm{r}-\mathrm{CN}$.

Fig. 1b. CN was completely dissolved in MSA, exhibiting a clear and yellow solution, and stirring would accelerate the dissolution speed (Fig. S5†). Different to $\mathrm{CN}$ nanosheets prepared by liquid-exfoliation, ${ }^{5 c}$ no evident Tyndall effect was observed for $\mathrm{CN}$ in MSA (Fig. S6a $\dagger$ ), indicating $\mathrm{CN}$ macromolecules of minimal lateral dimensions rather than nanosheets in MSA. As controls, CN only partially dissolved in concentrated $\mathrm{H}_{2} \mathrm{SO}_{4}$, ClSA, and EMS under the same conditions. Therefore, the dissolution ability of CN in the solvents followed the order MSA, $\mathrm{H}_{2} \mathrm{SO}_{4}$, ClSA and EMS.

To evaluate the possible changes of the electronic structure of $\mathrm{CN}$ after dissolution, $\mathrm{CN}$ was recovered from the MSA solution by using poor solvents (e.g. acetonitrile). As shown in Fig. 1c inset, the recovered $\mathrm{CN}$ ( $\mathrm{r}-\mathrm{CN}$ ) was still yellow, suggesting that $\mathrm{CN}$ did not disintegrate after dissolution. The UV-Vis spectrum of $\mathrm{r}-\mathrm{CN}$ (Fig. S7†) showed a minor blue-shift relative to that of CN. Such a blue-shift was also observed in the photoluminescence spectrum of r-CN (Fig. S8†), presumably due to the protonation of $\mathrm{CN}$ by MSA. ${ }^{3 a, 7}$

The molecular structure of r-CN was further studied by Fourier transform infrared spectroscopy (FT-IR) and X-ray photoelectron spectroscopy (XPS). As shown in Fig. 1c, for both $\mathrm{CN}$ and $\mathrm{r}-\mathrm{CN}$, the typical stretching mode of the $\mathrm{CN}$ heterocycles (1200-1600 $\mathrm{cm}^{-1}$ ) and the breathing mode $\left(810 \mathrm{~cm}^{-1}\right)$ were mostly retained. Broad and intense bands at 2800-3500 $\mathrm{cm}^{-1}$ and some peak-shifts were also observed, which might result from protonation by MSA. ${ }^{3 a, 7}$ The C 1s XPS spectra of r-CN and CN showed two main peaks at $288.3 \mathrm{eV}$ and $284.6 \mathrm{eV}$ (Fig. 1d), which are attributed to $\mathrm{N}-\mathrm{C}=\mathrm{N}$ and random adventitious carbon contamination, respectively. ${ }^{5 \boldsymbol{a}, \boldsymbol{b}, \mathbf{8}}$ Meanwhile, four $\mathrm{N} 1 \mathrm{~s}$ XPS peaks ascribed to $\mathrm{C}=\mathrm{N}-\mathrm{C}(398.2 \mathrm{eV}$, tri- $s$ triazine rings), $\mathrm{N}-(\mathrm{C}) 3$ (399.4 eV, bridging $\mathrm{N}$ atoms), $\mathrm{C}-\mathrm{N}-\mathrm{H}$ (400.9 eV, bonded with $\mathrm{H}$ atoms) and the charge effect were observed for both $\mathrm{CN}$ and r-CN (Fig. 1e), further verifying that these two compounds had similar $\mathrm{C}-\mathrm{N}$ bonding characteristics. $^{3 a}$ Elemental analysis (Table $\mathrm{S} 1 \dagger$ ) quantified the very similar molar $\mathrm{C} / \mathrm{N}$ ratios of $\mathrm{CN}(0.68)$ and $\mathrm{r}-\mathrm{CN}(0.67)$. The BET surface areas and pore volume of $\mathrm{CN}$ and $\mathrm{r}-\mathrm{CN}$ (Fig. S9†) were also not evidently changed. In addition, despite the fact that the precipitation recovery led to a lower crystallinity, the X-ray diffraction (XRD) pattern of $\mathrm{r}-\mathrm{CN}$ still exclusively demonstrated a similar predominant (002) diffraction peak to $\mathrm{CN}$, rather than peaks related to its intermediate precursors (Fig. $\mathrm{S} 2 \dagger$ ). All of these facts jointly demonstrated that the typical structure of $\mathrm{CN}$ was maintained after dissolution, rather than it being decomposed into smaller fragments.

To gain insight into MSA dissolving CN, we carried out firstprinciples density functional theory (DFT) calculations to analyze the adsorption sites, and adsorption abilities using a polarizable continuum model. The hollow $(\mathrm{H})$ position was found to be the most stable adsorption site relative to the top (T) and bridge (B) positions (Fig. 2a). Generally, a solvent molecule with a stronger adsorption ability could more competitively interact with $\mathrm{CN}$ and dissolve it. ${ }^{9}$ The calculated $E_{\text {ads }}$ values for MSA $(-0.84 \mathrm{eV}), \mathrm{H}_{2} \mathrm{SO}_{4}(-0.73 \mathrm{eV})$, and ClSA $(-0.67 \mathrm{eV})$ were all evidently higher than that for $\mathrm{H}_{2} \mathrm{O}(-0.27 \mathrm{eV})$, supporting the fact that the sulfonic acid group-containing solvents were more effective than $\mathrm{H}_{2} \mathrm{O}$ in dissolving $\mathrm{CN}$, and MSA was the best one in this study (Fig. 2b-d). Besides, because of the lack of protonation, the adsorption energy $(-0.14 \mathrm{eV})$ and distance $(2.38 \AA$ ) between the nearest $\mathrm{H}$ atom of EMS and the CN plane was apparently larger than that of the other three solvents, making EMS the poorest among them. Considering the contribution of entropy to the dissolution, the Gibbs energy was also calculated (see Computational methods in the ESI†), in agreement with the above results. This was also consistent with the recent computations for sulfonic acid group-containing surfactants in liquid-exfoliation of other layered materials. ${ }^{\mathbf{1 0}}$ (a)

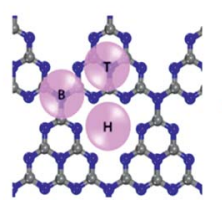

(b)

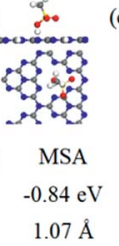

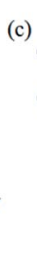

(d)

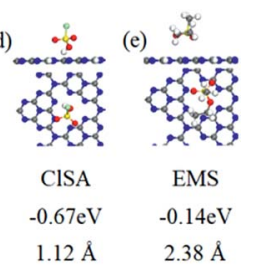

Fig. 2 (a) Structural model of a single CN layer for calculating the adsorption energies in different solvents. The insets show the typical adsorption sites: top $(\mathrm{T})$, hollow $(\mathrm{H})$, and bridge $(\mathrm{B})$. The most stable adsorption structures of MSA (b), $\mathrm{H}_{2} \mathrm{SO}_{4}$ (c), CISA (d) and EMS (e) molecules on $\mathrm{CN}$. $E_{\text {ads }}$ and $d_{\mathrm{H}-\mathrm{CN}}$ represent the adsorption energy and the distance between the nearest $\mathrm{H}$ atom and the $\mathrm{CN}$ plane, respectively. 
$\mathrm{CN}$ catalysts have been widely explored in heterogeneous conversion of organic molecules and harnessing solar energy to form chemical fuels; however, the activity was limited by low exposure of potential active sites. As a result, many attempts have been made, e.g. to prepare nanostructured $\mathrm{CN}$ with a higher density of exposed active sites. ${ }^{5,11}$ Driving a homogeneous catalytic reaction by $\mathrm{CN}$ at the molecular level is the ultimate goal. Taking advantage of the excellent dissolution of $\mathrm{CN}$ in $\mathrm{MSA}^{12}$ the photocatalytic dehydrogenation of $3,3^{\prime}, 5,5^{\prime}$ tetramethylbenzidine (TMB), a typical substrate for studies on enzymatic or biomimetic dehydrogenation oxidation (Fig. S10 $\dagger$ ), ${ }^{13}$ was firstly investigated. TMB was originally colorless in MSA and turned reddish brown after photocatalytic reaction; thus, the reaction rate could be quantitatively determined by using UV-Vis spectroscopy and the Beer-Lambert law when the concentration of oxidized TMB $\left(\mathrm{TMB}_{\mathrm{ox}}\right)$ was low.

The UV-Vis absorbance of the $\mathrm{TMB}_{\mathrm{ox}}$ in MSA increased dramatically with time when $\mathrm{CN}$ was used as a homogeneous catalyst (CN-homo, CN dissolved in MSA) under irradiation (Fig. 3a circles and Fig. S11†). In contrast, only negligible color changes were observed without $\mathrm{CN}$ or without light irradiation (Fig. 3a, triangles). Control experiments also showed that MSA itself did not promote the catalytic activity (Fig. S12 $\dagger$ ). These facts jointly indicated that the oxidation of TMB was exclusively driven by $\mathrm{CN}$-homo via a photocatalytic pathway. As a control, the same amount of $\mathrm{CN}$ was dispersed in an aqueous solution as a heterogeneous catalyst ( $\mathrm{CN}$-hetero) to drive the same reaction, but only very moderate activity was observed (Fig. 3a, squares). Although the blue-shift of CN-homo was not in favor of
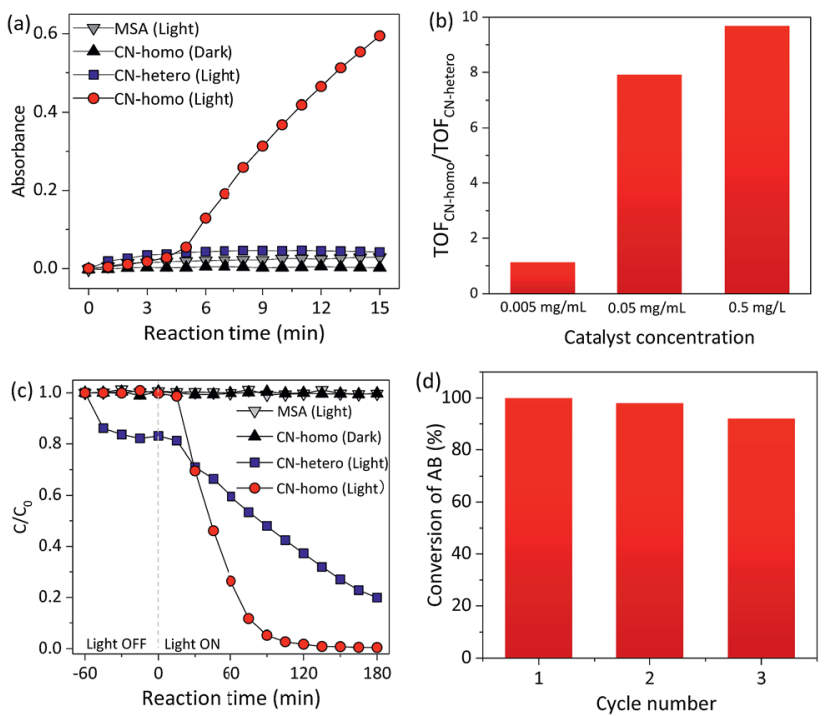

Fig. 3 (a) Absorbance of $\mathrm{TMB}_{\text {ox }}$ at $456 \mathrm{~nm}$ as a function of time during photocatalytic dehydrogenation using a homogeneous $\mathrm{CN}$ catalyst ( $\mathrm{CN}$-homo, CN dissolved in MSA) and heterogeneous $\mathrm{CN}$ catalyst (CNhetero, $\mathrm{CN}$ dispersed in $\mathrm{H}_{2} \mathrm{O}$ ). Catalyst loading: $0.5 \mathrm{mg} \mathrm{mL}^{-1}$ (see optimization in Fig. S12†). (b) Ratio of the TOFs for different concentrations of $\mathrm{CN}$-homo and $\mathrm{CN}$-hetero catalysts. (c) $C / C_{0}$ of $\mathrm{AB}$ concentration as a function of time during photocatalytic $\mathrm{N}$-demethylation using $\mathrm{CN}$-homo and $\mathrm{CN}$-hetero. (d) Re-use catalytic efficiency of the $\mathrm{CN}$-homo catalyst in the photocatalytic Ndemethylation of $A B$. improving the photocatalytic activity due to the eliminated absorbance of light, Fig. $3 \mathrm{~b}$ and $\mathrm{S} 13 \dagger$ still show that the turnover frequency (TOF) ${ }^{14}$ ratio of the $\mathrm{CN}$-homo system to the $\mathrm{CN}$-hetero system increased up to 10 -fold when the concentration of $\mathrm{CN}$ increased up to $0.5 \mathrm{mg} \mathrm{mL} \mathrm{mL}^{-1}$, demonstrating the inherent advantage of the $\mathrm{CN}$-homo in exposing active sites, especially at a high concentration, in driving a practical catalytic reaction. Notably, no activity was observed in the first several minutes for the CN-homo system, which may be attributed as an induction stage. $^{15}$

Recycling and reusability are challenging for homogeneous catalysts. ${ }^{16}$ Interestingly, this limitation did not happen for $\mathrm{CN}$ homo, because of the unique dissolution/recovery ability of $\mathrm{CN}$ in MSA. The collection yield of CN in MSA using diethyl ether as a poor solvent was up to $c a$. $95 \%$. To verify this assumption, in the second set of experiments, the CN-homo photocatalyst was used in the $N$-demethylation of Azure B (AB), which is an important chemical transformation in the pharmaceutical industry (Fig. S14 $\dagger$ ). ${ }^{17}$ In contrast to the darkening of TMB, the color of $\mathrm{AB}$ lightened after oxidation; thus, the Beer-Lambert law was valid for kinetic studies, especially when a high concentration of $\mathrm{CN}$ photocatalyst was used to eliminate the systematic loss of the catalyst during recycling.

Fig. $3 \mathrm{c}$ and $\mathrm{S} 15 \dagger$ show the variation in the $\mathrm{AB}$ concentration with reaction time. When $\mathrm{CN}$-homo was used, $\mathrm{AB}$ was almost completely oxidized (remnant $<5 \%$, see Fig. S16 and more discussion in the ESI $\dagger$ ) upon irradiation for $90 \mathrm{~min}$, while for $\mathrm{CN}$-hetero, more than $48 \%$ of unreacted $\mathrm{AB}$ remained, suggesting that the $\mathrm{CN}$-homo photocatalyst had a much faster oxidation rate. Control experiments without $\mathrm{CN}$ and without light irradiation (Fig. 3c, triangles) resulted in negligible activity within the prescribed time, confirming that the oxidation of $\mathrm{AB}$ was exclusively driven by $\mathrm{CN}$ via a photocatalytic pathway. The first-order rate constant (Fig. S17 $\dagger$ ) of the reaction by $\mathrm{CN}$-homo $\left(0.041 \mathrm{~min}^{-1}\right)$ was nearly six times that of the reaction driven by $\mathrm{CN}$-hetero (0.007 $\left.\mathrm{min}^{-1}\right)$. CN-homo was further explored in recycling experiments, in which the catalyst was separated by using diethyl ether as a poor solvent after the reaction and recycled in the next experiment. As shown in Fig. 3d, CN-homo maintained most of the original high photocatalytic activity for at least three cycles. The stability of the catalyst was also reflected in the similar XRD patterns of the CN after the photocatalytic reaction (Fig. S18 $)$ ). A minor deterioration in the catalytic activity was observed, presumably due to mechanical loss of the catalyst during the recycling process. Therefore, the recycling experiment demonstrated the unique features of the proposed $\mathrm{CN}$ photocatalytic system, which combines the advantages of both homogeneous catalysts that often exhibit superior activity, and heterogeneous catalysts that can be easily recycled and reused without evident activity loss. The catalytic activities of the dehydrogenation of TMB and the $N$-demethylation of $\mathrm{AB}$ were also compared using other (photo)catalysts (Tables S2 and S3 $\dagger$ ) and showed very competitive activities. Besides, the dissolved CN in MSA was also explored as a photocatalyst for water splitting, but no significant $\mathrm{H}_{2}$ evolution activity was observed. This may be caused by the fact that the current photocatalytic reaction conditions, e.g. the special 
nature of the MSA solvent and the protonated surface of $\mathrm{CN}$, were not favorable for $\mathrm{H}_{2}$ evolution. To overcome this issue, more studies are needed in the future.

\section{Conclusions}

In summary, $\mathrm{CN}$ was dissolved in environmentally friendly MSA at room temperature. The DFT results verified that the sulfonic acid group significantly reduced the adsorption energies. Taking the dehydrogenation of TMB and the $N$-demethylation of $\mathrm{AB}$ as examples, the homogeneous $\mathrm{CN}$ photocatalyst exhibited a dramatic enhancement in activity by a factor of up to 10 relative to the heterogeneous one due to the increase of potential accessible catalytic sites. Moreover, the reversible dissolution of CN using good/poor solvents allowed the homogeneous $\mathrm{CN}$ to be effectively recycled and reused, which united the advantages of both homo/heterogeneous catalysts. This work may extend the application of $\mathrm{CN}$ as a homogeneous (photo)catalyst and highlights the utility of polymeric catalysts with intrinsic catalytic properties in bridging the gap between homo/heterogeneous catalysis. Besides, the detailed structure information of dissolved $\mathrm{CN}$ in MSA is very interesting, and requires further experiments and molecular dynamics simulations in the future.

\section{Conflicts of interest}

There are no conflicts to declare.

\section{Acknowledgements}

This work was supported by the National Natural Science Foundation of China (21775018, 21675022), the Natural Science Foundation of Jiangsu Province (BK20160028, BK20170084), the Open Funds of the State Key Laboratory of Electroanalytical Chemistry (SKLEAC201703) and the Fundamental Research Funds for the Central Universities. We thank Prof. Shuxin Ouyang (Tianjin University, China) for helpful discussion.

\section{Notes and references}

1 (a) D. J. Cole-Hamilton, Science, 2003, 299, 1702; (b) A. Corma and H. Garcia, Top. Catal., 2008, 48, 8.

2 (a) X. C. Wang, K. Maeda, A. Thomas, K. Takanabe, G. Xin, J. M. Carlsson, K. Domen and M. Antonietti, Nat. Mater., 2009, 8, 76; (b) Y. Wang, X. C. Wang and M. Antonietti, Angew. Chem., Int. Ed., 2012, 51, 68; (c) S. W. Cao, J. X. Low, J. G. Yu and M. Jaroniec, Adv. Mater., 2015, 27, 2150; (d) R. Godin, Y. Wang, M. A. Zwijnenburg, J. Tang and J. R. Durrant, J. Am. Chem. Soc., 2017, 139, 5216; (e)
Z. Zhou, Y. Zhang, Y. Shen, S. Liu and Y. Zhang, Chem. Soc. Rev., 2018, 47, 2298.

3 (a) Z. X. Zhou, J. H. Wang, J. C. Yu, Y. F. Shen, Y. Li, A. R. Liu, S. Q. Liu and Y. J. Zhang, J. Am. Chem. Soc., 2015, 137, 2179; (b) J. S. Xu and M. Antonietti, J. Am. Chem. Soc., 2017, 139, 6026.

4 T. S. Miller, T. M. Suter, A. M. Telford, L. Picco, O. D. Payton, F. Russell-Pavier, P. L. Cullen, A. Sella, M. S. P. Shaffer, J. Nelson, V. Tileli, P. F. McMillan and C. A. Howard, Nano Lett., 2017, 17, 5891.

5 (a) P. Niu, L. Zhang, G. Liu and H.-M. Cheng, Adv. Funct. Mater., 2012, 22, 4763; (b) S. B. Yang, Y. J. Gong, J. S. Zhang, L. Zhan, L. L. Ma, Z. Y. Fang, R. Vajtai, X. C. Wang and P. M. Ajayan, Adv. Mater., 2013, 25, 2452; (c) X. D. Zhang, X. Xie, H. Wang, J. J. Zhang, B. C. Pan and Y. Xie, J. Am. Chem. Soc., 2013, 135, 18; (d) K. Schwinghammer, M. B. Mesch, V. Duppel, C. Ziegler, J. Senker and B. V. Lotsch, J. Am. Chem. Soc., 2014, 136, 1730. 6 N. Behabtu, J. R. Lomeda, M. J. Green, A. L. Higginbotham, A. Sinitskii, D. V. Kosynkin, D. Tsentalovich, A. N. ParraVasquez, J. Schmidt, E. Kesselman, Y. Cohen, Y. Talmon, J. M. Tour and M. Pasquali, Nat. Nanotechnol., 2010, 5, 406. 7 Y. Zhang, A. Thomas, M. Antonietti and X. Wang, J. Am. Chem. Soc., 2009, 131, 50.

8 (a) Q. Lin, L. Li, S. Liang, M. Liu, J. Bi and L. Wu, Appl. Catal., $B$, 2015, 163, 135; (b) W. Wu, J. Zhang, W. Fan, Z. Li, L. Wang, X. Li, Y. Wang, R. Wang, J. Zheng, M. Wu and H. Zeng, ACS Catal., 2016, 6, 3365.

9 B. Zhu, L. Zhang, B. Cheng and J. Yu, Appl. Catal., B, 2018, 224, 983.

10 M. Monajjemi, J. Mol. Liq., 2017, 230, 461.

11 (a) Q. Han, B. Wang, J. Gao and L. Qu, Angew. Chem., Int. Ed., 2016, 55, 10849; (b) K. S. Lakhi, D. H. Park, K. Al-Bahily, W. Cha, B. Viswanathan, J. H. Choy and A. Vinu, Chem. Soc. Rev., 2017, 46, 72.

12 M. D. Gernon, M. Wu, T. Buszta and P. Janney, Green Chem., 1999, 1, 127.

13 X. J. Cui, Y. H. Li, S. Bachmann, M. Scalone, A. E. Surkus, K. Junge, C. Topf and M. Beller, J. Am. Chem. Soc., 2015, 137, 10652.

14 F. Goettmann, A. Fischer, M. Antonietti and A. Thomas, Angew. Chem., Int. Ed., 2006, 45, 4467.

15 B. An, L. Zeng, M. Jia, Z. Li, Z. Lin, Y. Song, Y. Zhou, J. Cheng, C. Wang and W. Lin, J. Am. Chem. Soc., 2017, 139, 17747.

16 D. E. Bergbreiter, J. H. Tian and C. Hongfa, Chem. Rev., 2009, 109, 530.

17 G. B. Kok, C. C. Pye, R. D. Singer and P. J. Scammells, J. Org. Chem., 2010, 75, 4806. 